\title{
MAGNETIZED TYPE I ORBIFOLDS IN FOUR DIMENSIONS
}

\author{
Marianna Larosa \\ Dipartimento di Fisica, Università di Roma "Tor Vergata", INFN, Sezione di Roma II \\ Via della Ricerca Scientifica 1, 00133 Roma, Italy \\ Marianna.Larosa@roma2.infn.it \\ ROM2F-02/31
}

\begin{abstract}
I review the basic features of four dimensional $Z_{2} \times Z_{2}$ (shift) orientifolds with internal magnetic fields, describing two examples with $N=1 \mathrm{su}-$ persymmetry. As in the corresponding six-dimensional examples, D9branes magnetized along four internal directions can mimic $D 5$-branes, even in presence of multiplets of image branes localized on different fixed tori. Chiral low-energy spectra can be obtained if the model also contains $D 5$-branes parallel to the magnetized directions.
\end{abstract}

In the last few years, open-string models have considerably enlarged our view of consistent string vacua and have also acquired a valuable role for low-energy phenomenology. This is related to the presence of D-branes, where gauge and matter fields can be localized while gravity pervades the whole higher-dimensional bulk. Type I models can be described as orientifolds of the Type IIB string theory by the world-sheet parity transformation $\Omega$ [1], and compactifications on various toroidal orbifolds have also allowed for attractive brane supersymmetry (breaking) configurations [2, 3]. Two kinds of exactly solvable deformations can be introduced in Type I orbifold models: (continuous and discrete) Wilson lines and internal magnetic fields. Continous Wilson lines shift the momenta of open string states according to their charges, thus leading to gauge symmetry breaking while preserving the overall rank. In a Tdual picture, this effect can be ascribed to D-brane displacements in the ambient-space. On the other hand, discrete deformations (e.g. a quantized $B_{a b}$ ) lead in general to gauge group rank reductions. The effect of uniform magnetic fields $H_{i}$ introduced in compact internal space is also relatively simple, since they couple only to the string endpoints that, 
say, carry charges $q_{L}$ and $q_{R}$. The result, however, is different according to whether the total charge $Q=q_{L}+q_{R}$ is equal to or different from zero 画. In the latter case the oscillator frequencies are shifted, and there is a degeneracy in the Landau levels related to the non-commutativity of the string zero-modes. On the other hand, in the neutral case the oscillator modes are unshifted, but the zero-modes are affected, and involve rescaled (boosted) contributions to the one-loop annulus partition function. These magnetic deformations admit an alternative interpretation in terms of rotated branes [5]. In these types of constructions, related to magnetized branes by T-dualities, and widely analyzed in recent attempts to recover the Standard Model as a low-energy limit [6], chiral fermions typically lie at D-brane intersections. Thus, in this contest the need for multiple matter families translates into the requirement of multiple D-brane intersections. Furthermore, the introduction of magnetic fields provides an interesting way to break supersymmetry in Type I models [7], for particles of different spins couple differently to them via their magnetic moments, thus giving rise to different masses. For arbitrary configurations of the magnetic fields, the open spectrum is indeed non-supersymmetric, and tachyonic (Nielsen-Olesen) instabilities [8] are present. However, for (anti)self-dual magnetic field configurations [9], or, in the T-dual language, for particular intersection angles between the D-branes [5], tachyons are absent and some residual supersymmetry is preserved.

In the following I shall describe two classes of Type I magnetized models in four dimensions, built as open descendants of $Z_{2} \times Z_{2}$ (shift) orbifolds. In both cases the starting point is the Type IIB string theory compactified on a six-dimensional torus $T^{6}=T^{2} \times T^{2} \times T^{2}$, and projected by the $Z_{2} \times Z_{2}$ operations $o=(+,+,+), g=(+,-,-), h=(-,-,+)$ and $f=(-,+,-)$, that, aside from the identity $o$, act as $\pi$-rotations on two of the three internal tori. There are actually two classes of supersymmetric $Z_{2} \times Z_{2}$ models, that differ because of the possible presence of discrete torsion, a sign $(\epsilon= \pm 1)$ in their partition functions, with crucial effects both on the low-energy closed spectra and on the structure of the open descendants. Indeed, only models with discrete torsion $(\epsilon=-1)$ give rise to open descendants containing chiral fermions, but chirality in the open spectrum can also be recovered, for $\epsilon=1$, if suitable internal magnetic fields are introduced. Let us thus look more closely at models without discrete torsion. They contain a set of $D 9$-branes and three different sets of $D 5$-branes, $D 5_{1}, D 5_{2}$ and $D 5_{3}$. Homogeneous magnetic fields along two of the three two-tori alter the boundary conditions of the open strings stretched between these $D$-branes. Furthermore, in order to absorb the R-R charge of the background, the open sector contains also 
a set of magnetized D9-branes that, as in the six-dimensional examples of [9], mimic the behavior of one set of D5-branes. This is a brane transmutation: in a magnetic field, a D9-brane can acquire a D5 RR charge [10], and can therefore contribute to the corresponding tadpole. Chiral matter appears in the open spectrum when $D 5$-branes extend along the magnetized directions of the D9-branes, and typically arranges itself in multiple families. In a T-dual picture, the effect can be equivalently ascribed to the intersections of mutually rotated $D 7$-branes.

When the conventional orbifold operations are combined with momentum or winding shifts of the lattice states, the resulting models can display a partial breaking of supersymmetry, recovered for large or small radii, with peculiar $D 5$-brane configurations, that typically arrange themselves in multiplets of images, interchanged by some of the orbifold operations. This is what happens, for instance, for the $w_{2} p_{3}$ models of [11], with shifts of the lattice windings and momenta in the second and third internal tori. They have one set of $D 9$-branes and two sets of $D 5$-branes, $D 5_{1}$ and $D 5_{2}$, that appear organized in doublets and four-plets. The introduction of two magnetic fields in the second and third tori gives rise to a model with a chiral spectrum, as expected from the presence of the $D 5_{2}$ branes, that extend along a magnetized compact direction. Moreover, the brane transmutation phenomenon appears in this case in a subtler way, again because of the presence of $D 5$ multiplets. These are distributed among the orbifold fixed points, and thus it is not possible to insert all branes at a single fixed point. The D9-branes do their best to mimic the same behavior, even though they are not localized, owing to their spatial extension. However, the centers of the classical Landau orbits organize themselves in doublets as well, so that each orbit is accompanied by images, just like each $D 5$-brane in the undeformed model. Even in this case the open spectra reveal the presence of multiplets of matter families and an unusual rank reduction of the corresponding Chan-Paton group. An interesting by-product-of this analysis is that the familiar discrete deformations, induced by a quantized $B_{a b}$, can also be generated by suitable shift-orbifolds of this type. A more detailed discussion of these models may be found in [12, 13].

\section{Acknowledgments}

It is a pleasure to thank G. Pradisi for a stimulating collaboration and A. Sagnotti for introducing me to these topics. I am also grateful to the Organizers of the Cargèse 2002 ASI for giving me the opportunity to present these results. This work was supported in part by I.N.F.N., by the E.C. RTN programs HPRN-CT-2000-00122 and HPRN-CT-2000-00148, by the INTAS contract 99-1-590, by the MURST-COFIN contract 2001-025492 and by the NATO contract PST.CLG.978785. 


\section{References}

[1] A. Sagnotti, arXiv:hep-th/0208020; M. Bianchi and A. Sagnotti, Phys. Lett. B 247 (1990) 517, Nucl. Phys. B 361 (1991) 519; M. Bianchi, G. Pradisi and A. Sagnotti, Nucl. Phys. B 376 (1992) 365. For reviews see: E. Dudas, Class. Quant. Grav. 17 (2000) R41 [arXiv:hep-ph/0006190]; C. Angelantonj and A. Sagnotti, Phys. Rept. 371 (2002) 1 arXiv:hep-th/0204089.

[2] I. Antoniadis, E. Dudas and A. Sagnotti, Nucl. Phys. B 544 (1999) 469 arXiv:hep-th/9807011; I. Antoniadis, G. D'Appollonio, E. Dudas and A. Sagnotti, Nucl. Phys. B 553 (1999) 133 arXiv:hepth/9812118];

[3] E. Dudas and A. Sagnotti, Phys. Lett. B 464 (1999) 38 arXiv:hepth/9908023. C. Angelantonj, Nucl. Phys. B 566 (2000) 126 arXiv:hep-th/9908064]; G. Aldazabal and A. M. Uranga, JHEP 9910 (1999) 024 arXiv:hep-th/9908072.

[4] E. S. Fradkin and A. A. Tseytlin, Phys. Lett. B 163 (1985) 123; A. Abouelsaood, C. G. Callan, C. R. Nappi and S. A. Yost, Nucl. Phys. B 280 (1987) 599.

[5] M. Berkooz, M. R. Douglas and R. G. Leigh, Nucl. Phys. B 480 (1996) 265 arXiv:hep-th/9606139.

[6] M. Cvetic, G. Shiu and A. M. Uranga, Nucl. Phys. B 615 (2001) 3 arXiv:hep-th/0107166; R. Blumenhagen, B. Kors, D. Lust and T. Ott, Fortsch. Phys. 50 (2002) 843 arXiv:hep-th/0112015; R. Blumenhagen, V. Braun, B. Kors and D. Lust, arXiv:hepth/0210083.

[7] C. Bachas, arXiv:hep-th/9503030.

[8] N. K. Nielsen and P. Olesen, Nucl. Phys. B 144 (1978) 376.

[9] C. Angelantonj, I. Antoniadis, E. Dudas and A. Sagnotti, Phys. Lett. B 489 (2000) 223 hep-th/0007090; C. Angelantonj and A. Sagnotti, hep-th/0010279.

[10] M. R. Douglas, arXiv:hep-th/9512077; M. B. Green, J. A. Harvey and G. W. Moore, Class. Quant. Grav. 14 (1997) 47 arXiv:hepth/9605033.

[11] I. Antoniadis, G. D'Appollonio, E. Dudas and A. Sagnotti, Nucl. Phys. B 565 (2000) 123 hep-th/9907184.

[12] M. Larosa, arXiv:hep-th/0111187; G. Pradisi, arXiv:hepth/0210088.

[13] M.Larosa and G.Pradisi, in preparation. 